\title{
RICHARD THURNWALD 1869-1954
}

\section{Herbert Baldus}

Faleceram, quase ao mesmo tempo, as duas figuras mais representativas entre os etnólogos modernos de língua alemã. Richard Thurnwald morreu em 19 de janeiro de 1954 e o padre Wilhelm Schmidt no dia 10 do mês seguinte. Eram, também, quase da mesma idade. Aquêle nasceu em 18 de stembro de 1869, êste em 16 de fevereiro do ano anterior.

Ambos produziram, incessantemente, até o seu fim. Mas, os seus caminhos foram bem diversos. Schmidt nasceu na Vestfália e foi para a Áustria, onde criou a chamada "Escola de Viena". Thurnwald veio ao mundo em Viena e lecionou em Berlim. Embora ambos fôssem pesscas agradáveis e acessíveis, tinham caracteres essencialmente diversos e se mostraram antagonistas no campo da ciência. Uma prova disto é a crítica de Thurnwald à Teoria dos Ciclos Culturais e à Escola Histórico-Cultural ${ }^{1}$.

As linhas seguintes limitar-se-ão a dar algumas notas sôbre o mestre da universidade de Berlim e sua obra. Como pesquisador de campo $\mathrm{ccm}$ longos anos de experiência, Thurnwald frisou incansàvelmente a importância do empirismo, combatendo as orientações especulativas. Mas, nem por isso se contentou em descrever fatos e opô-los às hipóteses. Justamente seus últimos livros foram os que mais revelaram o pensador esforçado em obter uma visão geral do destino da humanidade ${ }^{2}$.

Desde que, em 1889, se matriculou na Universidade de Viena, Thurnwald sentiu-se atraído por problemas sociológicos. Mas, como naquele tempo não havia, ainda, cursos de sociologia e etnologia, resolveu estudar jurisprudência, economia e línguas orientais. Formouse em direito. Realizou sua primeira pesquisa de campo na Bósnia, investigando aspectos tradicionais de indústria e comércio.

Em 1901 começou a trabalhar no Museu Etnológico de Berlim. Foi esta instituição que o mandou, em 1906, para a Micronésia, o arquipélago de Bismarck e as ilhas de Salomão. Explorando estas regiões do oeste do Pacífico até 1909, Thurnwald reuniu não só coleções etnográficas, mas estudou a economia, língua, mitologia, organização social e outros aspectos das diversas culturas, bem como a psique dos indivíduos. Em 1912 foi para a Nova Guiné onde ficou até 1915. Subindo o rio Sepique, penetrou em terra incógnita cujos habitantes nunca tinham visto um homem branco. Um dos resultados mais conhecidos desta expedição é o trabalho sôbre a organização do parentesco dos Bánaro. 
Em 1930, Richard Thurnwald, acompanhado de sua espôsa, a etnóloga Hilde Thurnwald, seguiu para a África Oriental a fim de estudar a mudança cultural. Dois anos depois, ambos investigaram em Bougainville o que, a êsse respeito, sucedera aos indígenas desta ilha desde a visita que lhes fizera Richard Thurnwald em 1907.

Infelizmente permanecem inéditas, ainda, grandes partes dos resultados tanto desta última como das mencionadas outras expedições ao oeste do Pacífico. Fazemos votos para que a aluna e companheira do eminente pesquisador consiga publicá-los quanto antes.

Depois da primeira guerra mundial, Thurnwald ingressou no magistério universitário, lecionando, primeiro, em Halle, e, desde 1924, em Berlim as matérias de etnologia, sociologia e etno-psicologia. Quando, depois da segunda guerra mundial, elementos descontentes com a orientação dada à universidade berlinense, situada no setor de ocupação russa, fundaram uma "Universidade Livre", no setor de ocupação americana da antiga capital da Alemanha, Thurnwald passou para esta e aí continuou até morrer. Em diversas épocas de sua vida deu, também, cursos em várias universidades norte-americanas, isto é, na da Califórnia (Berkeley), de Yale, de Harvard e de Syracuse.

Thurnwald era bem o contrário de um mestre-escola modelar e de um orador brilhante. Mesmo na cátedra continuava introvertido, interrompendo, freqüentemente, a preleção a fim de tomar notas para uso próprio.

E' difícil definir sua obra com um simples rótulo. Thurnwald, o etnólogo, sociólogo, psicólogo, jurista e economista com trabalhos fundamentais em tôdas essas esperializações, é designado como "funcionalista" nos tratados metodológicos de Lowie e Mühlmann. Assim, no livro de Lowie, a segunda das duas partes do capítulo intitulado "Functionalism: pure and tempered" é uma apreciação crítica da obra de Thurnwald, frisando o caráter sociológico de sua orientação ${ }^{3}$.

Tratando dos representantes principais das tendências funcionalistas na etnologia, Mühlmann ${ }^{4}$ escreve a respeito das orientações características de Thurnwald: "Como sociólogo aperfeiçou, metòdicamente, o princípio de seleção biológica exigindo o estudo do peneiramento social; prosseguiu no desenvolvimento, portanto, da linha do antigo darwinismo social de Darwin e Spencer. Na psicologia social e étnica, Thurnwald é continuador de Bastian. Nas suas idéias sôbre problemas de evolução pode ser ligado, antes de tudo, a Tylor. Mas Thurnwald revela também, e pronunciadamente, orientação histórica e podese dizer que só êle cobre a lacuna deixada, com referência à etnologia, pela 'Escola Histórica' berlinense do século passado. Naturalmente, Thurnwald possui rica experiência de trabalho de campo. Distingue-se dos funcionalistas da Europa Ocidental, também, por não restringir a etnologia ao estudo dos povos-naturais, considerando, por princípio, todos os povos objeto de suas pesquisas. E' verdade que Thurnwald tem a particularidade de não designar seus trabalhos como 'etnológicos' quando tratam de povos 'superiores', mas como 'sociológicos'." 
O livro intitulado Beiträge zur Gesellungs- und Völkerwissenschaft (Berlin 1950) em que colaboraram amigos e antigos alunos de Thurnwald para prestar-lhe homenagem por ocasião de seu octogésimo aniversário, contém uma bibliografia de 353 publicações e 8 manuscritos inéditos do aniversariante.

Das obras publicadas destacam-se:

Forschungen auf den Salomo-Inseln und dem Bismarck-Archipel, 2 volumes, Berlin 1912, 538 e 92 pp.

Ethno-psychologische Studien an Südsee-Völkern. Leipzig 1913, 163 pp.

Die Gemeinde der Bánaro. Ehe, Verwandtschaft und Gesellschaftsbau eines Stammes im Innern von Nenguinea. Stuttgart 1921, 274 pp.

Psychologie des primitiven Menschen, Handbuch der vergleichenden Psychologie I, pp. 147-320, München 1922. e ss.

180 artigos in: M. Ebert: Reallexikon der Vorgeschichte, Berlin 1924, $48 \mathrm{pp}$.

Die Eingeborenen Australiens und der Südsee-Inseln, Tübingen, 1927,

Papuanisches und melanesisches Gebiet südlich des äquators einschliesslich Neuguinea, Das Eingeborenenrecht II, pp. 543-656, Stuttgart 1929 .

Koloniale Gestaltung. Methoden und Probleme überseeischer Ausdehnung. Hamburg 1939, 492 pp.

Völkerkunde von Afrika, Essen 1940, xv, $665 \mathrm{pp}$. (em colaboração com H. Baumann e D. Westermann).

Der Mensch geringer Naturbeherrschung. Sein Aufstieg zwischen Vernunft und Wahn. Berlin 1950, $157 \mathrm{pp}$.

A obra-prima de Thurnwald é Die menschliche Gesellschaft in ihren ethno-soziologischen Grundlagen (A Sociedade Humana em suas bases etno-sociológicas). Seu primeiro volume intitulado "Repräsentative Lebensbilder von Naturvölkern" (Berlin \& Leipzig, 1931, xxiii, 311 pp.) apresenta, depois de longa introdução sôbre problemas gerais da etnologia e sua história, uma série de resumidas descrições da vida e cultura dos povos-naturais de diversas partes do mundo: captores, agricultores e pastores, a título de exemplificação. O segundo volume (1932, viii, 360 pp.), "Werden, Wandel und Gestaltung von Familie, Verwandtschaft und Bünden im Lichte der Völkerforschung", trata da organização social pròpriamente dita. "Werden, Wandel und Gestaltung der Wirtschaft im Lichte der Völkerkunde" é o título do terceiro volume (1932, vii, 248 pp.) dedicado ao estudo da economia. O quarto volume (1935, xix, 377 pp.) chama-se "Werden, Wandel und Gestaltung von Staat und Kultur im Lichte der Völkerkunde", tratando, depois de definir numerosos termos técnicos, dos problemas de origem e desenvolvimento das organizações políticas, bem como dos conceitos de "cultura" e "civilização", "progresso" e "ciclo" e outros assuntos que mais atrairam o teórico em Richard Thurnwald. Talvez contenha êste quarto volume os elementos que mais caracterizam a personalidade científica do autor. O quinto volume (1934, viii, 232 pp.), intitulado "Werden, Wandel und Gestaltung des Rechtes im Lichte der Völkerkunde", "deve ser considerado fundamental para o estudo das origens do direito", como Emílio Willems escreveu prefaciando sua versão portuguêsa". 
Convém chamar a atenção para a repetição das palavras "Werden, Wandel und Gestaltung" ("desenvolvimento, mudança e formação") nos títulos dos volumes II, III, IV e V, pois revelam a importância dada por Thurnwald aos aspectos dinâmicos em todos os objetos de suas pesquisas.

A bibliografia publicada em 1950 menciona como sendo o VI volume da Sociedade Humana o manuscrito de um "ensaio de uma paleopsicologia". Este trabalho apareceu impresso, em 1951, sob o título Des Menschengeistes Erwachen, Wachsen und Irren, Versuch einer PaIäopsychologie von Naturvölkern, mas sem a declaração expressa de ser parte da referida obra monumental. Thurnwald pretendia dar com êle "uma história do pensamento" (p. 10).

Não figuram naquela bibliografia os seguintes artigos: "Eine Meinung zum Drama der Menschheit", SCIENTIA, junho de 1953; "Sippe und Stamm", Handwörterbuch der Sozialwissenschaften, Stuttgart 1953, pp. 272-277; "Der Wandel der Erscheinungen und Gedanken des Zusammenlebens", SOCIOLOGUS N. S., I, pp. 8-31, II, pp. 26-52, Berlin 1951-52. Tão pouco é mencionada a nova série do SOCIOLOGUS, importante revista dirigida por Thurnwald e por êle fundada, em 1925, sob o título ZEITSCHRIFT FƯR VÖLKERPSYCHOLOGIE UND SOZIOLOGIE, deixando de existir em 1933 com a supressão da liberdade do pensamento na Alemanha e ressuscitando em 1951 como publicação em alemão inglês.

Os estudiosos que não sabem alemão, podem entrar em contacto direto com as idéias de Thurnwald pelas suas publicações seguintes:

\section{Em inglês:}

Bánaro Society. Social Organization and Kinship System of a Tribe in the Interior of New Guinea. Mem. Am. Anthr. Asso., III, pp. 251-391. 1916. Social Problems of Africa. Africa 1929, pp. 130-136.

Social Systems of Africa. Africa 1929, pp. 221-242.

4 artigos in: Encyclopaedia of the Social Sciences, New York 1930 e s. The Missionary's Concern in Sociology and Psychology. Africa 1931, pp. 418-433.

Economics in Primitive Communities. London 1932, 314 pp.

Sociology: Its Content and Application. Social Forces XI, pp. 161-17., 1932.

Social Transformations in East Africa. Am. Jr. of Sociol. XXXVIII, pp. 175-184, 1932 .

The Psychology of Acculturation. Am. Anthropologist XXXIV, pp. 557$569,1932$.

The Missionary's Anthropological Research. Int. Inst. of Afr. Languages \& Cultures, Memor. VIII, London 1932, 31 pp. (em colaboração com Westermann) .

The Social Function of Personality. Sociology and Soc. Res., XVII, pp. 203-218, 1933.

Some Traits of Society in Melanesia. Proc. 5th Pacific Sc. Congr., Victoria and Vancouver, British Columbia, 1933, pp. 2805-2814, 1934.

Adventures of a Tribe in New Guinea (the Tjimundo). Essays presented to C. G. Seligman. London 1934, pp. 345-360. 
Stone Monuments in Buin. Oceania V, pp. 214-217, Sydney 1934.

Pigs and Currency in Buin. Observations about Primitive Standards of Value and Economics. Oceania, V. pp. 119-141, Sydney 1934.

Black and White in East Africa. London 1935, 409 pp. 1936 .

Studying Savages in Melanesia. The Yale Rev., XXXVI, pp. 313-332,

The Price of the White Man's Peace. Pacific Affairs, IX-3, 1936, $11 \mathrm{pp}$.

Profane Literature of Buin. Yale Univ. Publ. in Anthropology, VIII, New Haven, 1936, 15 pp.

The Crisis of Imperialism in East Africa and elsewhere. Social Forces, XV, pp. 84-91, 1936.

Contributions toward Analysis of the Mechanism of Culture. I: Civilization and Culture. Am. Sociol. Rev., I, pp. 387-395, 1936. - II: Progress Viewed as a Component in the Configuration of Culture. Am. Sociol. Rev., I, fp. 604-613, 1936. - III : Cultural Rotation, Its Propulsion and Rhythm. Am. Sociol. Rev., II, pp. 26-42, 1937.

The African in Transition. Some Comparisons with Melanesia. Africa XI, pp. 174-186, 1939 .

\section{Em francês:}

Direction et séthisation comme problème socio-psychologique. Congr. Internat. de Psychologie Appliquée. Paris 1929.

Un questionnaire psycho-physio-morphologique pour l'étude de la psychologie des races. Revue Anthropologique, XL, pp. 262-277, 1930 . (em colaboração com F. H. C. van Loon).

L'Économie Primitive. Préface et traduction de Charles Mourey. Bibliothèque Scientifique. Payot, Paris 1937. $390 \mathrm{pp}$.

L'Esprit Humain: éveil, croissance et tatonnements. Essai de Paléopsychologie. Traduction d'André Coeuroy. Bibliothèque Scientifique. Payot, Paris 1953. $344 \mathrm{pp}$.

\section{Em português:}

Numerosos verbetes do Dicionário de Etnologia e Sociologia de Baldus e Willems contêm versões de definições e comentários dados por Thurnwald em várias de suas obras, principalmente na Sociedade Humana. Aliás, um retrato de Thurnwald encontra-se pegado ao frontispício daquele Dicionário. A revista SOCIOLOGIA publicou em continuações, do n. 3 do vol. III até o n. 1-2 do vol. VII (São Paulo 1941-1945), em tradução de Emílio Willems intitulada "Origem, formação e transformação do Direito à luz das pesquisas etnológicas" as primeiras 130 páginas do quinto volume da Sociedade Humana.

Richard Thurnwald, um dos maiores inspiradores da etnologia moderna, era um guia de seus discípulos pessoais, sempre lhes aplanando os caminhos. Mesmo na idade avançada continuou amigo, sempre cuidando dos interesses dos amigos. Pensou também no Brasil. Foi um dos primeiros a inscrever-se como membro do XXXI Congresso Internacional de Americanistas a ser realizado na capital de São Paulo, em agôsto de 1954 . 
(1) Versão portuguêsa no Dicionário de Etnologia e Sociologia de Baldus e Willems, São Paulo 1939, pp. 49-54.

(2) Cf. as minhas resenhas na Revista do Museu Paulista, N. S., vol. V, São Paulo 1951, pp. 264-265, e vol. VI, 1952, pp. 516-517.

(3) Robert H. Lowie: The History of Ethnological Theory, New York 1937, pp. 242-249.

(4) Wilhelm Mühimann: Methodik der Völkerkunde, Stuttgart 193s, p. 162 .

(5) SOCIOLOGIA, vol. III, n. 3, São Paulo 1941, p. 238.

(6) Duncker \& Humblot, Berlin, $448 \mathrm{pp}$. 